\title{
Culpabilidad, resistencia y autonomía: respuesta a Guillermo Hurtado
}

\author{
Óscar MARTIARENA \\ Facultad de Filosofía y Letras \\ Universidad Nacional Autónoma de México \\ martifyl@servidor.unam.mx
}

\begin{abstract}
Resumen: El texto es una respuesta a la recensión crítica que Guillermo Hurtado hace del libro Culpabilidad y resistencia de Óscar Martiarena. En su respuesta, Martiarena precisa los objetivos de su investigación, básicamente orientada a estudiar algunas de las formas a través de las cuales los indios mexicanos se convirtieron en "objetos" por conocer para los misioneros españoles. En particular, Martiarena señala que en su trabajo busca mostrar cómo, a través de la práctica de la confesión sacramental, los indios fueron "objetivados" y, con ello, reconocidos por los misioneros como culpables y resistentes a la práctica confesional. Asimismo, en su texto, Martiarena responde otras cuestiones presentadas por Hurtado tocantes al uso de la noción de autonomía en Culpabilidad y resistencia.
\end{abstract}

Palabras clave: subjetividad, conciencia individual, confesión, idolatría

Debo expresar, ante todo, mi más sincero agradecimiento a Guillermo Hurtado por su atención hacia mi trabajo de indagación sobre las formas en las que se intentó constituir modos específicos de subjetividad entre los indios de la Nueva España. Debo también decir que leer sus comentarios y sostener conversaciones con él me alientan en la continuación del trabajo emprendido del que espero, en verdad, conduzca a conclusiones que nos permitan, tal y como Hurtado señala, "revisar no sólo nuestra visión de la evangelización [...], sino algo más amplio y hondo, a saber, la historia misma de la subjetividad en México".

En relación con sus comentarios a mi libro Culpabilidad y resistencia, considero pertinente hacer algunas precisiones sobre el contenido de mi trabajo y la dirección que sigue. Tales precisiones, aunque amplias, me parecen necesarias en tanto que considero que Hurtado hace una lectura apresurada de mi libro, la cual lo lleva a no considerar elementos que en el propio texto se muestran adecuadamente, a pasar por alto cuestiones que el texto abre y a echar de menos problemas que en su redacción mi libro no se propuso resolver.

Así, de principio, debo señalar que mi trabajo, tanto en el artículo "El indio como objeto de conocimiento", publicado en el volumen correspondiente a 1998 de Diánoia y mencionado por Hurtado, como en Culpabilidad y resistencia, se ha orientado a indagar fundamentalmente cómo es que los 
indios mexicanos se convirtieron, desde los inicios mismos de la evangelización, en "objetos" por conocer para los misioneros españoles. Ahora bien, en ambos trabajos, más que asumir en definitiva como "verdaderas" las descripciones presentes en las crónicas elaboradas por misioneros de distintas órdenes, entre las cuales la de fray Bernardino de Sahagún está en primer lugar, me pareció pertinente preguntarme por los motivos que los cronistas tuvieron para convertir a los habitantes del Nuevo Mundo en objetos de conocimiento y la forma en que se construyó la "verdad" sobre los indios de la Nueva España, la cual con frecuencia se acepta sin mediar crítica alguna. En particular, en "El indio como objeto de conocimiento", la investigación se orientó a intentar precisar: 1) los objetivos que tenía Sahagún al estudiar a los indios; 2) la procedencia del método de investigación utilizado por el franciscano, y 3) el "marco conceptual" que le permitió a Sahagún emprender su empresa de conocimiento. Al respecto, las conclusiones obtenidas son: que Sahagún buscaba conocer a los indios con el fin de extirpar las prácticas indígenas ajenas a lo que el Occidente cristiano aceptaba como convenientes; que el método de investigación utilizado por Sahagún tiene elementos comunes con las prácticas inquisitoriales, y que el "marco teórico" del franciscano estaba constituido fundamentalmente por conceptos propios de la metafísica medieval. Ahora bien, ha de advertirse que, en la investigación realizada, el objeto de estudio fue básicamente la Historia general de las cosas de Nueva España y que lo fue en la dirección de los puntos señalados. De la investigación emprendida me pareció oportuno concluir que, en su empresa, Sahagún convirtió al indio, más que en un ser semejante a todos los seres humanos, en objeto de conocimiento.

En Culpabilidad y resistencia, la investigación tiene búsquedas similares a las del artículo aludido; es decir, me aboqué también a estudiar la forma en que los indios fueron "objetivados", aunque ahora la indagación se orientó a estudiar una práctica específica de objetivación de particular importancia para los evangelizadores: la confesión auricular. Fruto de siglos de desarrollo, la confesión era parte integral del cristianismo que se dio a la tarea de conquistar espiritualmente a los habitantes del Nuevo Mundo. Ahora bien, como intento mostrar en el primer capítulo del libro, la confesión se había consolidado como la forma fundamental de penitencia cristiana y un instrumento de gran eficacia en la catequización, en tanto que promovía en cada uno de los fieles el ejercicio de un riguroso examen de sí mismo a la luz de prescripciones claramente establecidas por la Iglesia, una severa autovigilancia en torno a lo que las propias prescripciones establecían y una verbalización de todos sus pecados. Con ello, la práctica de la confesión se convirtió en un instrumento de individualización de los fieles a los que la Iglesia inducía a constituir una conciencia individual de sí mismos; pero conviene subrayar que la conciencia de sí a la que inducía la Iglesia en 
cada uno de los fieles tiene la característica de realizarse bajo el signo de la culpa; es decir, la inducida por la Iglesia de fines de la Edad Media e inicios de la Modernidad no es cualquier tipo de conciencia individual, sino una conciencia individual culpable, dado que los preceptos de la Iglesia conducían a que los fieles se reconocieran a sí mismos, en todo momento de su vida, como individuos culpables.

El acercamiento a la confesión en el horizonte europeo del siglo XVI realizado en el primer capítulo del libro, análisis fundamental para pensar en la atención que los evangelizadores pusieron en la implantación de su práctica entre los indios, me permitió detenerme en las crónicas y otros documentos que refieren su introducción y normalización en la Nueva España. Ciertamente, al examinar las crónicas franciscanas se tiene la impresión de que la confesión fue incorporada rápidamente en el ámbito indígena. Sin embargo, conviene tener en cuenta la discrepancia entre los relatos de franciscanos y lo que otros documentos muestran en torno a la "fácil" incorporación de los indios al sacramento. Al optimismo de Motolonía, que franciscanos posteriores reproducen, se opone la opinión de agustinos y dominicos, las ordenanzas de los Concilios Provinciales que buscan regular "desviaciones" de la práctica confesional y las observaciones hechas en diversas doctrinas publicadas en lenguas castellana y mexicana sobre el significado y la relevancia del sacramento. En particular, en relación con la "difícil" incorporación de los indios a la práctica de la confesión, destaca la preocupación de Sahagún, quien emprende su gran obra con el fin de que confesores y predicadores accedieran al conocimiento de las prácticas idolátricas indias que debían ser abolidas, particularmente a través de la predicación y la confesión, las cuales, de acuerdo con Sahagún, los evangelizadores debían realizar con un conocimiento adecuado de los indios. ${ }^{1}$ Ahora bien, lo que el análisis de las crónicas, doctrinas, documentos de los Concilios Provinciales y la preocupación de Sahagún revela, es la dificultad de que los indios aceptaran el sacramento y lo realizaran tal y como debía ser. Es decir, contra el parecer de Motolinía, que en su crónica se muestra "optimista" sobre la confesión sacramental de los indios, lo que otros documentos revisados muestran es que los religiosos tenían serias dificultades al querer implantar la práctica confesional entre los indios. Por su parte, la lectura de las crónicas tardías de los siglos XVII y XVIII muestra dificultades constantes en la confesión de los indios. Los textos se refieren continuamente a los indios como rústicos, carentes de culpa y memoria y de cortedad de entendimiento. Ahora bien, en esta dirección, lo que revela

\footnotetext{
${ }^{1}$ En el segundo capítulo del libro menciono algunas noticias sobre lo que algunos cronistas, entre ellos Sahagún, refieren como "confesión indígena". Al respecto, remito al lector a la lectura de la nota 51 del capítulo, en la que expongo las razones por las cuales se deben tomar con cautela los relatos en los que los cronistas asocian prácticas indígenas con la confesión cristiana.
} 
el análisis de los documentos referidos es claro. Lo que se va construyendo en los diversos documentos estudiados es la imagen de un indio que es culpable por su condición de mortal, pero que además es incapaz de culpabilizarse por su propia naturaleza de indio.

En el capítulo tercero del libro, en un primer momento, emprendo la descripción y el análisis de Manuales de confesión y Sumas para confesores publicados en la Nueva España durante los siglos XVI, XVII y XVIII, entre los que destacan el manual de Alonso de Molina de 1569 y el de Juan Bautista de 1599, sin duda los más completos, particularmente en relación con su similitud con los manuales europeos de la época. Al introducirnos en los editados en la Nueva España, llama la atención la exhortación que ambos franciscanos dirigen a los indios a fin de que, antes de cualquier otra consideración, asuman su culpabilidad, lo cual cada indio habría de hacer a través de un examen de conciencia, el cual debería realizar a la luz de diversas prescripciones que los franciscanos presentan. Cabe añadir que en el resto de ambos manuales se mantiene el tono culpabilizador con el que el penitente debe asumirse como pecador. Y en este sentido, lo que se muestra en los confesionarios de los franciscanos es la pretensión de conformar entre los indios una conciencia individualizada cuyo punto de referencia debería ser la consideración, por parte de cada uno de los indios, de su condición de individuo culpable.

En la segunda parte del tercer capítulo se analizan las principales $\mathrm{Su}$ mas para confesores publicadas en la Nueva España que, como las europeas, tenían el objetivo de proporcionar a los curas de almas bases para formular juicios precisos acerca de los pecadores y administrar correctamente el sacramento de la penitencia. En particular, las Sumas novohispanas enfrentan diversos casos específicos relacionados con las dificultades en la administración del sacramento. De acuerdo con las Sumas, los casos recurrentes sobre la confesión de los indios son los siguientes: los indios acuden al confesionario sin culpa alguna y, por consiguiente, no se muestran arrepentidos; no tienen memoria de sus pecados; no saben hacer su examen de conciencia, todo lo cual se explica porque los indios son gente rústica, de corto entendimiento, de corta capacidad, torpes, rudos y no conocen la doctrina. A los argumentos señalados, el agustino Manuel Pérez añade, en 1713, que los indios son, además, ladinos, "hábiles" para eludir la confesión, se la pasan en la taberna pecando, y cuando se les pregunta por sus pecados, "se atarantan y no dicen culpa alguna", amén de ser "incapaces" de realizar una buena confesión.

Pero, además de las dificultades para una correcta confesión, las Sumas consignan, aunque también lo hacen algunos manuales de confesión, la preocupación de los confesores por las prácticas que llaman "idolátricas" realizadas continuamente por los indios. Por su persistencia en sus "idola- 
trías", diversos ministros novohispanos aseguran que los indios "disimulan" frente a los españoles y en "secreto" realizan sus ritos en fechas específicas que recomiendan a todo cristiano conocer a fin de denunciar a los idólatras. En suma, los confesores novohispanos añaden a la condición de culpables de los indios, la de resistentes a asumirse como buenos cristianos; es decir, la condición de resistentes a abandonar sus prácticas, necesariamente idolátricas para los confesores en tanto no eran conformes con los rituales cristianos.

Habiendo hecho una descripción de la dirección y del contenido de mi trabajo, paso a responder puntualmente los comentarios de Guillermo Hurtado.

1. Lo que los evangelizadores buscaban al introducir diversas prácticas propias del cristianismo entre los indios era convertirlos en "buenos cristianos". Una de esas prácticas, la confesión, era fundamental para sus propósitos, dada la importancia que tenía para el cristianismo del siglo XVI, en especial después del cisma protestante y el consiguiente Concilio de Trento que redobló esfuerzos por generalizar la práctica confesional en el ámbito del catolicismo. La primera parte de mi libro la dedico a estudiar la procedencia de la confesión auricular, sus características y la manera en que los teólogos se esforzaron en fundamentarla; particularmente, en el apartado 1.10, "Una interpretación: individualidad y penitencia", desarrollo un acercamiento a la forma de conciencia que genera la práctica confesional que conceptualmente llamé 'conciencia individual culpable', dado que, como he expuesto antes, los preceptos de la Iglesia conducían a que los fieles se reconocieran a sí mismos, en todo momento de su vida, como individuos culpables. Así, no digo, como sugiere Hurtado, que los evangelizadores tenían como propósito "crear y apuntalar" tal tipo de conciencia entre los indios, sino lo que afirmo es que la práctica de la confesión auricular como tal genera una conciencia individual culpable. Mi tesis principal tampoco es, como señala Hurtado, que los indios se resistieron de "una manera muy hábil a la culpabilización que les era demandada por los confesores". Lo que afirmo en el libro es que el optimismo inicial de Motolinía en relación con la incorporación de los indios al sacramento, retomado en términos similares por otros franciscanos posteriores, puede ser cuestionado a la luz de lo que otras crónicas relatan, de las regulaciones establecidas por los Concilios Provinciales, de lo que se señala en diversas doctrinas y de la preocupación de Sahagún en torno a la permanencia de diversas prácticas idolátricas entre los indios. Más aún, en mi libro busco mostrar que el optimismo inicial de los franciscanos es rebatido por los términos en los que los manuales de confesión y las sumas para confesores fueron escritos del siglo XVI al XVIII en la Nueva España. En tales documentos, como se desprende de las precisiones antes expuestas, los indios son vistos por los 
confesores novohispanos como culpables por su propia condición de mortales, y, con ello, objetivados; pero además por no aceptar, ni reconocer, ni arrepentirse de sus culpas; y como resistentes, en tanto que no realizan adecuadamente su examen de conciencia y no abandonan sus prácticas idolátricas. En esta dirección, la investigación presentada en Culpabilidady resistencia se mantiene en la misma dirección que "El indio como objeto de conocimiento", pues trata de mostrar diversas formas en las que los indios fueron "objetivados" durante la evangelización. Por ello, lejos de coincidir, como sostiene Hurtado, con la aseveración del agustino Manuel Pérez, para quien los indios, además de rústicos y ladinos, eran hábiles para eludir la confesión y se la pasaban pecando en la taberna, lo que me interesa mostrar es cómo fueron objetivados y, con ello, reconocidos e identificados por los confesores novohispanos. En este sentido, debo insistir en que lo que pretende mi trabajo es precisamente mostrar la forma en que a lo largo de casi tres siglos los indios fueron objetivados, particularmente a través de los textos que la implantación de una práctica como la confesión sacramental generó en la Nueva España. Y en esta dirección, lo que revelan los textos es que los indios fueron objetivados por los confesores novohispanos como rústicos, de corto entendimiento, carentes de memoria, incapaces para la confesión, que no saben contar, indolentes, ladinos, idólatras, evasivos; en suma, culpables y resistentes a la práctica de la confesión. Y éste es precisamente el objetivo y ésta la tesis fundamental de mi libro: demostrar que un análisis de los textos del siglo XVI al XVIII publicados en la Nueva España, en los que se habla de la confesión de los indios, revela que fueron objetivados como culpables y resistentes. Con la exposición de los textos y la discusión en torno a ellos, me parece que la tesis sobre la forma en que los indios fueron objetivados a través de la práctica de la confesión queda demostrada.

2. Por lo indicado, queda claro que la discusión que emprende Hurtado en relación con dos supuestas versiones de la tesis de mi libro y las hipótesis alternativas que presenta no son pertinentes. Salvo una brevísima alusión a las investigaciones de Alfredo López Austin, en mi libro no me propongo realizar una indagación minuciosa acerca de la conciencia que tenían los indios antes de la llegada de los españoles. ${ }^{2}$ No obstante, Hurtado señala

\footnotetext{
${ }^{2}$ En relación con la conciencia que tenían los indios dentro de su propia cultura, me limito a hacer una breve alusión en la p. 161 del libro, en donde señalo: "En suma, tal y como los confesionarios europeos de la época lo hacían, los elaborados por los misioneros franciscanos buscaban la conformación de un tipo de individualidad muy particular entre los indios. Individualidad que, al parecer, era sensiblemente diferente a la que las comunidades indias tenían antes de la llegada de los españoles. De acuerdo con los estudios de Alfredo López Austin, lo que privó en las sociedades prehispánicas fue un tipo de representación en la cual los indios se conciben a sí mismos como pertenecientes a la comunidad antes que a sí mismos. El individuo se representa a sí mismo en función del lugar que ocupa en su grupo
} 
que las bases probatorias que doy para referirme a la forma de la conciencia de sí en los indios no son suficientes. Pero debo decirle, y con ello dejar clara la cuestión, que no era el objetivo de mi libro, ni mucho menos pretendía dirigir hacia allá su tesis principal, dar cuenta de la conciencia de sí que tenían los indios, sino, insisto, analizar y describir la forma en la que fueron objetivados, frente a la cual se les exigía dar respuestas precisas y concretas y asumirse tal y como los confesores novohispanos lo demandaban. Lo que me interesa mostrar enfáticamente en el libro es que, de acuerdo con los documentos analizados, la práctica de la confesión exigía una forma de conciencia específica en los indios y que, al no obtener lo esperado, al no encontrar en los indios una identidad como la que esperaban, los confesores optaron por identificarlos, como menciono al final del libro, mediante un estigma que los reducía de antemano. Así, no es veraz, como dice Hurtado, que sólo he "contado la mitad de la historia". Lo que he intentado hacer es mostrar la forma en que los confesores novohispanos objetivaron a los indios en relación con la práctica de la confesión y, con ello, hacer una descripción minuciosa de la estigmatización de la que fueron objeto. Ahora bien, quizá sea factible pensar, como sugiere Hurtado, en la posibilidad de que algún tipo de investigación intente dar cuenta de lo que los indios pensaban en torno a la práctica de la confesión. Sin embargo, el problema fundamental al respecto es que la conquista espiritual, al tiempo que exigía en los indios una individualidad precisa y los estigmatizaba, se ocupó de reprimir la memoria indígena. Y en esta dirección, a falta de documentos históricos, debo decir que en algunos fragmentos del libro, básicamente al final, inferí resistencia a la confesión por parte de los indios a partir de las propias descripciones de los confesores. No obstante, a diferencia de los ministros novohispanos, me pareció plausible conceder a los indios una especie de "principio de caridad", como sugiere D. Davidson. Es decir, decidí pensar que las actitudes de los indios en el confesionario y las relacionadas con la preservación de sus rituales obedecían a cierta racionalidad. Ciertamente, con ello me situé por encima de lo que pensaban los confesores novohispanos. Para ellos no había racionalidad en las actitudes de los indios ni tampoco en sus prácticas, a las que llamaban "idolátricas", porque exigían una forma de racionalidad previamente determinada. Al no encontrarla, los confesores novohispanos declararon a los indios inútiles, rústicos, sin memoria, de corto entendimiento; esto es, irracionales. El confesor exigía una subjetividad que no encontraba en el indio. Pero bien

social que, a su vez, es un sitio en el cosmos al que debe respetar y cuidar. Es decir, en la sociedad prehispánica el indio tiene una conciencia y una memoria de sí mismo en función de la casta a la que pertenece. Lo cual significa no que no tenga conciencia ni memoria, sino que la tiene con relación a su comunidad y el lugar que en ella ocupa." Dentro del libro, es la única referencia que hago a la conciencia de los indios, pero de ninguna manera pretendo con ello dar cuenta de lo que merece una investigación específica. 
podríamos preguntar si acaso la respuesta de los indios carecía totalmente de racionalidad; al menos, el continuar con sus rituales, tal y como señalan los manuales de confesión y las sumas para confesores, puede pensarse como algo racional. Ahora bien, a esta respuesta de los indios, más allá de la percepción de los ministros novohispanos, se la puede llamar resistencia. $\mathrm{Y}$ en esta dirección debo decir que mi libro, al intentar mostrar la forma en que los indios fueron estigmatizados sin que los ministros novohispanos otorgaran importancia a sus culturas, pretende abrir un espacio desde el cual sea posible pensar que, ahí donde los confesores de indios no encontraron más que falta de talento, incapacidad y rusticidad, existía cierto tipo de conciencia, de memoria, de subjetividad, de valores que los indios no querían abandonar; abrir un espacio desde el cual sea lícita la pregunta acerca del lugar desde el cual los indios no aceptaron constituirse como conciencias individuales culpables, tal y como les exigía una práctica como la confesión sacramental. Incluso, abrir un espacio desde el que hoy podamos preguntarnos sobre las prácticas a través de las cuales se ha objetivado a los indios.

3. En relación con el tercer señalamiento de Hurtado, lo que digo en la página 62 del libro es que una práctica como la confesión sacramental inducida por la Iglesia a fines de la Edad Media y principios de la Modernidad no contribuye a la formación de un individuo autónomo, entendido como aquel que obedece una ley autoimpuesta. Básicamente, porque la confesión sacramental conduce a la formación de lo que denomino una 'conciencia individual culpable', caracterizada como una conciencia que "de ninguna manera contribuye a la formación de un individuo autónomo, ni valora su singularidad o independencia frente a su grupo". Debo añadir que tal tipo de conciencia es producto de las demandas de la Iglesia y no de una decisión autónoma del individuo. Así que mi planteamiento no es, como dice Hurtado, que un sujeto con conciencia individual culpable no pueda valorarse a sí mismo, ni su individualidad, sino que tal valoración, en el caso de la confesión sacramental y del examen de conciencia correspondiente exigido por la Iglesia en el tiempo histórico del que me ocupo, no es autónoma, dado que no es producto de una ley autoimpuesta por el propio individuo, sino de la aplicación de una ley establecida en forma heterónoma por la Iglesia. El concepto de 'conciencia individual culpable' que presento en mi libro camina en esta dirección, más allá del hecho ciertamente posible de que un individuo decida autónomamente valorar su conducta a través de preceptos que lo culpabilizan a cada instante. Pero en el momento histórico al que me refiero, los individuos que se sujetaban a la práctica de la confesión sacramental no lo hacían autónomamente, dado que la Iglesia, que funcionaba públicamente, los conminaba a conformar una memoria individual y una interpretación de sí mismos que los culpa- 
bilizaba en todo momento, que los obligaba, bajo amenaza de condena eterna, a revisar cada uno de sus pensamientos, palabras y obras bajo el signo de la culpa. No olvidemos que si la Ilustración, en términos de Kant, apuesta por la autonomía de los individuos bajo el lema "iTen el valor de valerte de tu propio entendimiento!", lo hace precisamente en contra de la tutela religiosa que quiere gobernar la conciencia de los individuos, impidiendo con ello su autonomía. En breve, no es que una conciencia autónoma no pueda culpabilizarse y regirse a través de la culpa, sino que la conciencia individual culpable, tal como la definí en mi libro en relación con el periodo histórico al que me refiero, es en principio heterónoma en la medida en que es una imposición externa al individuo. Me parece que con esto respondo a las preguntas que Hurtado me formula en relación con el concepto de conciencia individual culpable propia de la confesión vinculado con la noción kantiana de autonomía. Ahora bien, en el apartado final de la primera parte del libro parto, en efecto, de la noción de autonomía en el sentido kantiano. Tal noción me sirve de marco para ensayar una interpretación sobre el grado de "autonomía" de los individuos en algunas de las diversas formas históricas de la penitencia cristiana. En este sentido, la noción utilizada es un telón de fondo con el que pretendo hacer la interpretación mencionada. He de insistir en que, al evaluar la confesión auricular, se puede observar que no se caracteriza por generar individuos autónomos. Por otra parte, en el último párrafo de las conclusiones de Culpabilidad y resistencia hago referencia a la actual búsqueda de autonomía de los pueblos indios en México, particularmente porque una práctica como la confesión auricular de suyo no la proporciona incluso a los indios actuales. En esta dirección, coincido desde luego con Hurtado en que a los indios de la Nueva España, una autonomía como la kantiana les era negada tanto por los confesores novohispanos como por sus propias culturas. Asimismo, coincido con Hurtado cuando señala que en muchas de las comunidades que actualmente exigen autonomía, hoy en día apenas hay espacio para la tolerancia y la pluralidad. Pero, en este sentido, me parece que el problema apenas se abre. Es decir, quizá convenga pensar y discutir en las formas de autonomía que exigen algunos grupos indígenas. Al respecto, señalaría que la última aseveración de mi libro sólo pretende, con lo andado, sugerir que una práctica como la confesión sacramental no es ni puede ser fuente de la autonomía hoy anhelada.

4. Agradezco nuevamente la delicadeza que ha tenido Guillermo Hurtado al presentarme sus comentarios sobre mi trabajo. Sólo añadiría que mis investigaciones, más que concebirse como una mera historia de las mentalidades, tienen otras pretensiones: tal como sugiere Hurtado, mi propósito es revisar nuestra visión de la evangelización y la historia de nuestra subjetividad, tal vez para pensar de otra manera, para pensarnos en forma di- 
ferente. Michel Foucault, de quien soy deudor en mis acercamientos, decía al respecto de sus trabajos de "historia": "Se trata de un ejercicio filosófico: en él se ventila saber en qué medida el trabajo de pensar su propia historia puede liberar al pensamiento de lo que piensa en silencio y permitirle pensar de otro modo." 3

Recibido el 14 de enero de 2002; aceptado el 19 de marzo de 2002

${ }^{3}$ M. Foucault, Historia de la sexualidad II. El uso de los placeres, 8a. ed., México, Siglo XXI, 1996, p. 12. 